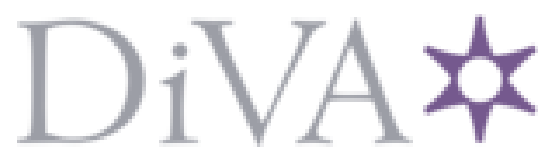

http://www.diva-portal.org

This is the published version of a paper published in Physical Review A. Atomic, Molecular, and Optical Physics.

Citation for the original published paper (version of record):

Lundh, E., Martikainen, J. (2012)

Kelvin-Helmholtz instability in two-component Bose gases on a lattice.

Physical Review A. Atomic, Molecular, and Optical Physics, 85(2): 023628

http://dx.doi.org/10.1103/PhysRevA.85.023628

Access to the published version may require subscription.

N.B. When citing this work, cite the original published paper.

Permanent link to this version:

http://urn.kb.se/resolve?urn=urn:nbn:se:umu:diva-53258 


\title{
Kelvin-Helmholtz instability in two-component Bose gases on a lattice
}

\author{
E. Lundh ${ }^{1}$ and J.-P. Martikainen ${ }^{2,3}$ \\ ${ }^{1}$ Department of Physics, Umea University, 90187 Umeå, Sweden \\ ${ }^{2}$ Nordita, 10691 Stockholm, Sweden \\ ${ }^{3}$ Aalto University, P.O. Box 15100, FI-00076 Aalto, Finland
}

(Received 9 November 2011; published 23 February 2012)

\begin{abstract}
We explore the stability of the interface between two phase-separated Bose gases in relative motion on a lattice. Gross-Pitaevskii-Bogoliubov theory and the Gutzwiller ansatz are employed to study the short- and long-time stability properties. The underlying lattice introduces effects of discreteness, broken spatial symmetry, and strong correlations, all three of which are seen to have considerable qualitative effects on the Kelvin-Helmholtz instability. Discreteness is found to stabilize low flow velocities because of the finite energy associated with displacing the interface. Broken spatial symmetry introduces a dependence not only on the relative flow velocity but also on the absolute velocities. Strong correlations close to a Mott transition will stop the Kelvin-Helmholtz instability from affecting the bulk density and creating turbulence; instead, the instability will excite vortices with Mott-insulator-filled cores.
\end{abstract}

DOI: 10.1103/PhysRevA.85.023628

PACS number(s): 03.75.Mn

\section{INTRODUCTION}

The Kelvin-Helmholtz instability is a dynamical instability of the interface between two fluids that move relative to one another. This hydrodynamical instability can occur in very different settings, for example, when the wind is blowing over the surface of the smooth ocean or in plasma flows in the Earth's magnetic field [1] or at the $A B$ phase boundary in superfluid ${ }^{3} \mathrm{He}[2,3]$. The creation of degenerate mixtures of weakly interacting different bosonic gases [4-6] enables studies of the Kelvin-Helmholtz instability in quantum systems that are more controllable as well as easier to measure in detail than more strongly interacting superfluids such as liquid ${ }^{3} \mathrm{He}$. Takeuchi and Suzuki et al. [7,8] performed theoretical studies of the Kelvin-Helmholtz instability in a two-component condensate. Essentially, Ref. [7] confirmed the expectation that Bose-Einstein-condensed atomic gases provide an ideal setting to study the classic Kelvin-Helmholtz instability without viscosity complicating the picture. Reference [8] found a considerably altered instability dispersion relation in the case of a wide interface with large overlap between the two condensates; this was classified as a countersuperflow instability.

Putting a Bose gas in an optical lattice introduces several features. Most dramatically, the gas exhibits a quantum phase transition between a superfluid and a Mott-insulating state when the ratio between the tunneling and interaction energies passes a critical value [9]. In a binary condensate the phase diagram is richer, displaying different combinations of Mott insulator and superfluid in coexisting or phase-separated configurations [10,11]. Moreover, even in the superfluid state where a significant portion of the atoms are Bose-Einstein condensed, the discreteness and the broken translational symmetry can have a decisive effect on the motion of the bosons, as we shall see.

In this paper, we study the Kelvin-Helmholtz-type instabilities of a phase-separated binary condensate on a lattice. In Sec. II, we present the equations that we work with. In Sec. III A, we study a weakly segregated pair of condensates in order to explore the effects of broken translational symmetry on the interface dynamics. In Sec. III B, we study instead a strongly segregated pair of condensates to see the effects of discreteness of the lattice. Section IV presents a heuristic derivation of a closed-form expression for the numerically obtained instabilities. In Sec. V, we study the system close to a Mott transition in order to explore the effects of strong correlations. Finally, in Sec. VI, we summarize and conclude.

\section{THEORY OF A TWO-COMPONENT LATTICE BOSE GAS}

The system is assumed to consist of two species of bosons at nearly zero temperature hopping on a square lattice, as can be realized with evaporatively cooled atoms in an optical lattice [4-6]. If the lattice is deep enough, the tight-binding approximation is valid, and the bosons can be assumed to occupy the lowest band only. For simplicity, we assume the two species to have equal masses, interaction constant, and tunneling properties; this can be realized by choosing the two components to be two spin states of the same element. The many-body Hamiltonian governing this system is

$$
\begin{aligned}
\hat{H}= & -\sum_{j=1}^{2} \sum_{\left\langle r r^{\prime}\right\rangle} J_{j} \hat{a}_{j r}^{\dagger} \hat{a}_{j r^{\prime}} \\
& +\frac{1}{2} \sum_{j=1}^{2} U_{j j} \sum_{r} \hat{a}_{j r}^{\dagger} \hat{a}_{j r}^{\dagger} \hat{a}_{j r} \hat{a}_{j r} \\
& +U_{12} \sum_{r} \hat{a}_{1 r}^{\dagger} \hat{a}_{1 r} \hat{a}_{2 r}^{\dagger} \hat{a}_{2 r}-\sum_{j=1}^{2} \mu_{j} \sum_{r} \hat{a}_{j r}^{\dagger} \hat{a}_{j r} .
\end{aligned}
$$

Here $J_{j}$ are the tunneling matrix elements, $U_{j j}$ are the in-species interaction parameters, and $U_{12}$ is the interspecies interaction parameter. $\mu_{j}$ is the chemical potential of species $j$. The summation index $j=1,2$ denotes the different species, the index $r$ runs over the lattice sites, and $\sum_{\left\langle r r^{\prime}\right\rangle}$ indicates a nearest-neighbor sum. We will be studying a two-dimensional square lattice in this paper, and we therefore pass to a vector 
notation, $\mathbf{r}=(x, z)$, where we let the dimensionless Cartesian coordinates $x$ and $z$ take on integer values.

For strong enough hopping and for high enough density, the lattice gases are almost entirely Bose-Einstein condensed, and the problem can be treated using Gross-Pitaevskii and Bogoliubov analysis. This regime is suitable for identifying the effects of the discreteness and broken translational invariance of the lattice, effects that do not depend on quantum fluctuations. In this regime, the lattice gas is accurately described using condensate wave functions $\Phi_{j}(\mathbf{r}, t)=\left\langle\hat{a}_{j r}\right\rangle$, whose dynamics follows from the discrete two-component Gross-Pitaevskii (GP) equation:

$$
\begin{aligned}
i \frac{\partial \Phi_{1}(\mathbf{r}, t)}{\partial t}= & -J_{1} \nabla^{2} \Phi_{1}(\mathbf{r}, t) \\
& +\left[U_{11}\left|\Phi_{1}\right|^{2}+U_{12}\left|\Phi_{2}\right|^{2}\right] \Phi_{1}(\mathbf{r}, t), \\
i \frac{\partial \Phi_{2}(\mathbf{r}, t)}{\partial t}= & -J_{2} \nabla^{2} \Phi_{2}(\mathbf{r}, t) \\
& +\left[U_{22}\left|\Phi_{2}\right|^{2}+U_{12}\left|\Phi_{1}\right|^{2}\right] \Phi_{2}(\mathbf{r}, t),
\end{aligned}
$$

where the discrete Laplacian is defined as

$$
\nabla^{2} \Phi(\mathbf{r})=\sum_{\mathbf{r}^{\prime}} \Phi\left(\mathbf{r}^{\prime}\right)-4 \Phi(\mathbf{r}),
$$

and the sum over $\mathbf{r}^{\prime}$ runs over the nearest neighbors to the site $\mathbf{r}$. We added the $-4 \Phi(\mathbf{r})$ term for convenience so that the Laplacian of a constant is zero, as one would usually expect. This amounts only to a shift in the energy scale.

\section{A. Bogoliubov approach}

In the Bogoliubov approximation one assumes for each component a stationary wave function with a small timedependent perturbation, $\delta \Psi_{i}(\mathbf{r}, t)$. We further assume that each condensate moves along the $x$ axis and has thus an associated plane-wave factor with a wave vector $k_{i}$. The wave functions are then given by

$$
\begin{aligned}
& \Phi_{1}(\mathbf{r}, t)=\left[\Psi_{1}(z) e^{i k_{1} x}+\delta \Psi_{1}(\mathbf{r}, t)\right] \exp \left(-i \mu_{1} t\right), \\
& \Phi_{2}(\mathbf{r}, t)=\left[\Psi_{2}(z) e^{i k_{2} x}+\delta \Psi_{2}(\mathbf{r}, t)\right] \exp \left(-i \mu_{2} t\right) .
\end{aligned}
$$

The equation of motion to zeroth order is the timeindependent one-dimensional (1D) GP equation that is used to calculate the interface profile,

$$
\begin{gathered}
-J_{1} \frac{\partial^{2} \Psi_{1}(z)}{\partial z^{2}}+\left[U_{11}\left|\Psi_{1}(z)\right|^{2}+U_{12}\left|\Psi_{2}(z)\right|^{2}\right. \\
\left.+2 J_{1}\left(1-\cos k_{1}\right)\right] \Psi_{1}(z)=\mu_{1} \Psi_{1}(z), \\
-J_{2} \frac{\partial^{2} \Psi_{2}(z)}{\partial z^{2}}+\left[U_{22}\left|\Psi_{2}(z)\right|^{2}+U_{12}\left|\Psi_{1}(z)\right|^{2}\right. \\
\left.+2 J_{2}\left(1-\cos k_{2}\right)\right] \Psi_{2}(z)=\mu_{2} \Psi_{2}(z),
\end{gathered}
$$

where we introduced notation analogous to Eq. (3),

$$
\frac{\partial^{2} \Psi_{j}(z)}{\partial z^{2}}=\Psi_{j}(z+1)-2 \Psi_{j}(z)+\Psi_{j}(z-1) .
$$

At this level, for a fixed atom number, the condensate flow has actually no effect on the condensate density profiles since a nonzero wave vector only amounts to an effective shift of the chemical potentials. However, this shift must be included to derive correct Bogoliubov equations.

Anticipating that the nonlinear term in the GP equation will couple positive- and negative-frequency modes, we define in a standard way

$$
\delta \Psi_{i}(\mathbf{r}, t)=\left[u_{i}(\mathbf{r}, t) \exp (-i \omega t)+v_{i}^{*}(\mathbf{r}, t) \exp (i \omega t)\right] .
$$

Ignoring the second-order terms in the perturbations we find the Bogoliubov equations for the discrete double condensate system,

$$
\hat{M} \mathbf{v}=\omega \hat{\eta} \mathbf{v},
$$

where

$\hat{M}=\left(\begin{array}{cccc}H_{1} & U \Psi_{1}^{2} e^{2 i k_{1} x} & C^{\prime} & D^{\prime} \\ U \Psi_{1}^{* 2} e^{-2 i k_{1} x} & H_{1} & D^{\prime *} & C^{\prime *} \\ C^{\prime *} & D^{\prime} & H_{2} & U \Psi_{2}^{2} e^{2 i k_{2} x} \\ D^{\prime *} & C^{\prime} & U \Psi_{2}^{* 2} e^{-2 i k_{2} x} & H_{2}\end{array}\right)$,

$$
\hat{\eta}=\left(\begin{array}{cccc}
1 & 0 & 0 & 0 \\
0 & -1 & 0 & 0 \\
0 & 0 & 1 & 0 \\
0 & 0 & 0 & -1
\end{array}\right),
$$

and

$$
\mathbf{v}=\left(\begin{array}{l}
u_{1}(\mathbf{r}) \\
v_{1}(\mathbf{r}) \\
u_{2}(\mathbf{r}) \\
v_{2}(\mathbf{r})
\end{array}\right)
$$

The coefficients of the matrix $\hat{M}$ are defined through $\quad C^{\prime}=U_{12} \Psi_{1}(z) \Psi_{2}(z) \exp \left[i\left(k_{1}-k_{2}\right) x\right], \quad D^{\prime}=$ $U_{12} \Psi_{1}(z) \Psi_{2}(z) \exp \left[i\left(k_{1}+k_{2}\right) x\right], n_{i}=\left|\Psi_{i}(z)\right|^{2}$,

$$
H_{1}=-J_{1} \nabla^{2}+2 U_{11} n_{1}+U_{12} n_{2}-\mu_{1},
$$

and

$$
H_{2}=-J_{2} \nabla^{2}+2 U_{22} n_{2}+U_{12} n_{1}-\mu_{2} .
$$

Let us repeat some common terminology and standard results [12]: The norm of a Bogoliubov mode is defined as

$$
\|\mathbf{v}\|=\sum_{r} \mathbf{v}^{\dagger}(\mathbf{r}) \hat{\eta} \mathbf{v}(\mathbf{r})
$$

Unless the norm is zero, we are free to rescale the Bogoliubov vector so that its absolute value is unity. A mode with $\|\mathbf{v}\|=1$ is called a quasiparticle mode, and a mode with $\|\mathbf{v}\|=-1$ is called a quasihole mode. If $\Psi$ is the ground state, then all the quasiparticle modes have positive eigenfrequencies, and all quasihole modes have negative eigenfrequencies; otherwise, no such rule applies. A mode with complex eigenfrequency has zero norm. Such modes will grow exponentially in time, and thus they signal a dynamical instability. We will therefore be interested in the imaginary parts of the Bogoliubov eigenfrequencies in this paper.

The symmetries of the problem give rise to degeneracies in the Bogoliubov spectrum. First, the well-known quasiparticlequasihole symmetry of the Bogoliubov equations [12] carries over to the two-component case: Each real eigenvalue $\omega$ 
with an eigenvector $\mathbf{v}=\left(u_{1}(\mathbf{r}), v_{1}(\mathbf{r}), u_{2}(\mathbf{r}), v_{2}(\mathbf{r})\right)^{T}$ has a corresponding solution with an eigenvalue $-\omega$ and an eigenvector

$$
\mathbf{v}=-\left(v_{1}(\mathbf{r})^{*}, u_{1}(\mathbf{r})^{*}, v_{2}(\mathbf{r})^{*}, u_{2}(\mathbf{r})^{*}\right)^{T} .
$$

In addition, imaginary eigenvalues appear in pairs of $\omega$ and $\omega^{*}$. Since we will be mostly interested in a symmetric interface where $\Psi_{1}(\mathbf{r})=\Psi_{2}(-\mathbf{r})$, we have an additional degenerate solution

$$
\left(u_{2}(-\mathbf{r}), v_{2}(-\mathbf{r}), u_{1}(-\mathbf{r}), v_{1}(-\mathbf{r})\right)^{T},
$$

with eigenvalue $\omega$. Combining Eqs. (13) and (14), we obtain

$$
\left(v_{2}^{*}(-\mathbf{r}), u_{2}^{*}(-\mathbf{r}), v_{1}^{*}(-\mathbf{r}), u_{1}^{*}(-\mathbf{r})\right)^{T},
$$

with eigenvalue $-\omega$. The latter two symmetries hold strictly only when equal and opposite currents are induced in the two components, as we will discuss further below.

\section{One moving condensate}

The discrete system is not translationally invariant, and we can therefore not use the relative velocity of the condensates as a unique parameter; also the absolute individual currents are physically relevant. In particular, we will study two different cases: the case where only one of the condensates carries a current and that where the condensates support equal but counterflowing currents.

We first assume that component 2 is moving along the $x$ direction and that all other position dependence of the stationary solutions is along $z$. This means that $\left(k_{1}, k_{2}\right)=(0, k)$ and

$$
H_{2}=-J_{2} \nabla^{2}+2 U_{22} n_{2}+U_{12} n_{1}-\mu_{2}^{(0)}-2 J_{2}(1-\cos k),
$$

where $\mu_{i}^{(0)}$ is the chemical potential in the absence of a current; the term $2 J_{2}(1-\cos k)$ due to the current is made explicit for convenience. The excitations can be expanded as plane waves along the $x$ direction. Dependency on the $x$ coordinate is eliminated with the choice

$$
\begin{aligned}
& u_{1}(\mathbf{r})=u_{1}(z) \exp \left(i k_{e} x-i k x / 2\right), \\
& v_{1}(\mathbf{r})=v_{1}(z) \exp \left(i k_{e} x-i k x / 2\right), \\
& u_{2}(\mathbf{r})=u_{2}(z) \exp \left(i k_{e} x+i k x / 2\right), \\
& v_{2}(\mathbf{r})=v_{2}(z) \exp \left(i k_{e} x-3 i k x / 2\right) .
\end{aligned}
$$

The Bogoliubov equations are now reduced to a $1 \mathrm{D}$ problem, and the Bogoliubov matrix takes the form

$$
\hat{M}_{1 \mathrm{D}}=\left(\begin{array}{cccc}
H_{1 u} & U \Psi_{1}^{2} & C & D \\
U \Psi_{1}^{* 2} & H_{1 v} & D^{*} & C^{*} \\
C^{*} & D & H_{2 u} & U \Psi_{2}^{2} \\
D^{*} & C & U \Psi_{2}^{* 2} & H_{2 v}
\end{array}\right),
$$

where the diagonal elements are

$$
\begin{aligned}
H_{1 u}= & H_{1 v}=-J_{1} \frac{\partial^{2}}{\partial z^{2}}+2 J_{1}\left[1-\cos \left(k_{e}-k / 2\right)\right] \\
& +2 U_{11} n_{1}+U_{12} n_{2}-\mu_{1}^{(0)}, \\
H_{2, u_{2}}= & -J_{2} \frac{\partial^{2}}{\partial z^{2}}-2 J_{2}(1-\cos k)+2 J_{2}\left[1-\cos \left(k_{e}+k / 2\right)\right] \\
& +2 U_{22} n_{2}+U_{12} n_{1}-\mu_{2}^{(0)},
\end{aligned}
$$

$$
\begin{aligned}
H_{2, v_{2}}= & -J_{2} \frac{\partial^{2}}{\partial z^{2}}-2 J_{2}(1-\cos k)+2 J_{2}\left[1-\cos \left(k_{e}-3 k / 2\right)\right] \\
& +2 U_{22} n_{2}+U_{12} n_{1}-\mu_{2}^{(0)},
\end{aligned}
$$

and $C=U_{12} \Psi_{1}(z) \Psi_{2}(z), D=U_{12} \Psi_{1}(z) \Psi_{2}(z)$ are now only functions of $z$.

\section{Counterdirected currents}

In the case of equal and opposite currents in the respective components, we have $\left(k_{1}, k_{2}\right)=(k / 2,-k / 2)$. Analogously to the last section, the excitations can be written as

$$
\begin{aligned}
& u_{1}(\mathbf{r})=u_{1}(z) e^{i k_{e} x+i k x / 2}, \\
& v_{1}(\mathbf{r})=v_{1}(z) e^{i k_{e} x-i k x / 2}, \\
& u_{2}(\mathbf{r})=u_{2}(z) e^{i k_{e} x-i k x / 2}, \\
& v_{2}(\mathbf{r})=v_{2}(z) e^{i k_{e} x+i k x / 2} .
\end{aligned}
$$

We find that the off-diagonal terms of the matrix $\hat{M}_{1 \mathrm{D}}$ are the same as before, but to the diagonal we get

$$
\begin{aligned}
H_{1, u_{1}}= & -J_{1} \frac{\partial^{2}}{\partial z^{2}}-2 J_{1}(1-\cos k / 2)+2 J_{1}\left[1-\cos \left(k_{e}+k / 2\right)\right] \\
& +2 U_{11} n_{1}+U_{12} n_{2}-\mu_{1}^{(0)}, \\
H_{1, v_{1}}= & -J_{1} \frac{\partial^{2}}{\partial z^{2}}-2 J_{1}(1-\cos k / 2)+2 J_{1}\left[1-\cos \left(k_{e}-k / 2\right)\right] \\
& +2 U_{11} n_{1}+U_{12} n_{2}-\mu_{1}^{(0)}, \\
H_{2, u_{2}=} & -J_{2} \frac{\partial^{2}}{\partial z^{2}}-2 J_{2}(1-\cos k / 2)+2 J_{2}\left[1-\cos \left(k_{e}-k / 2\right)\right] \\
& +2 U_{22} n_{2}+U_{12} n_{1}-\mu_{2}^{(0)}, \\
H_{2, v_{2}=} & -J_{2} \frac{\partial^{2}}{\partial z^{2}}-2 J_{2}(1-\cos k / 2)+2 J_{2}\left[1-\cos \left(k_{e}+k / 2\right)\right] \\
& +2 U_{22} n_{2}+U_{12} n_{1}-\mu_{2}^{(0)} .
\end{aligned}
$$

Expressed in the reduced 1D Bogoliubov amplitudes $u_{j}(z)$ and $v_{j}(z)$, the symmetries (13)-(15) acquire new forms since the effective Hamiltonians appearing in the diagonal of the Bogoliubov eigenvalue problem are different. The symmetry of greatest interest to us is that, for a given $k$ and $k_{e}$, each real eigenvalue $\omega$ with a reduced eigenvector $\left(u_{1}(z), v_{1}(z), u_{2}(z), v_{2}(z)\right)^{T}$ now has a corresponding hole mode with eigenvalue $-\omega$ and reduced eigenvector

$$
\left(v_{2}(-z), u_{2}(-z), v_{1}(-z), u_{1}(-z)\right)^{T} \text {. }
$$

\section{KELVIN-HELMHOLTZ INSTABILITY IN A DISCRETE SYSTEM}

After assuming that the two condensates have equal chemical potentials $\mu_{1}=\mu_{2}=\mu$, tunneling $J_{1}=J_{2}=J$, and interaction strengths $U_{11}=U_{22}=U$, the system can be characterized by three parameters. These are chosen to be the ratios $J / U$ and $U_{12} / U$ and the number density per site far from the interface $\bar{n}$. The chemical potential $\mu$ can then be calculated for given $J, U_{12}$, and $\bar{n}$. In addition, the problem is characterized by the wave vectors for the flow in each of the components, $\left(k_{1}, k_{2}\right)$. In Sec. II, we derived the detailed form of the Bogoliubov equations for one moving 


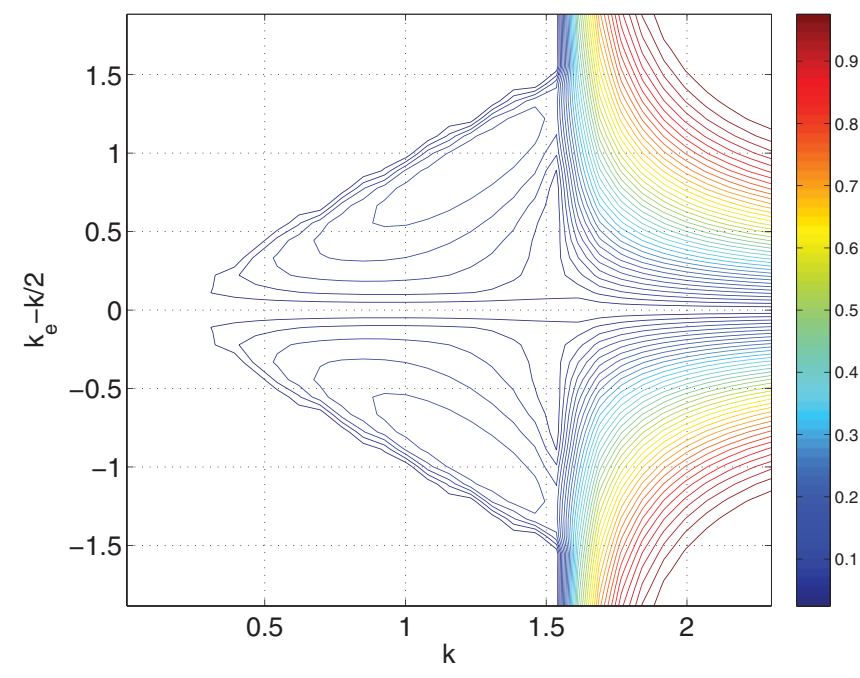

FIG. 1. (Color online) Growth rate of the dynamically most unstable mode (i.e., the imaginary part of the mode with the largest imaginary frequency) as a function of flow wave vector $k$ and and mode wave vector $k_{e}$ (note that a shift $k_{e}-k / 2$ has been applied to the $y$ axis to make the symmetry around $k_{e}=k / 2$ more transparent). Here one component is in motion with wave vector $k$ and the other is stationary. The lattice has 256 sites, the parameter values are $J / U=1, U_{12} / U=1.1$, and the asymptotic density is $\bar{n}=1$.

condensate, $\left(k_{1}, k_{2}\right)=(0, k)$, and opposite currents, $\left(k_{1}, k_{2}\right)=$ $(k / 2,-k / 2)$.

\section{A. Weak coupling and broken translational symmetry}

We first consider the case $J / U=1, U_{12} / U=1.1, \bar{n}=1$. Figure 1 shows the stability properties of the system in the case of one moving condensate, $\left(k_{1}, k_{2}\right)=(0, k)$. Figure 1 plots the largest imaginary excitation frequency as a function of flow wave vector $k$ and excitation wave vector $k_{e}$; thus, a vertical cross section in Fig. 1 at a fixed $k$ gives the imaginary part of the spectrum for a given current. The lattice is of finite size, 256 sites, in the numerics (in fact, real-life lattices may be of similar dimensions), and this influences the numerical value of the imaginary part somewhat. The effect is such that the imaginary part becomes larger as the lattice size is reduced to 128. The physics at low wave vector $k$ is clearly recognized from the case of a pair of classical fluids or a homogeneous two-component Bose-Einstein condensate (BEC) [7,8,13,14]. For a given $k$, not too large, a range of excitations with wave vectors $0<\left|k_{e}-k / 2\right| \lesssim k$ becomes unstable. The amplitudes of the unstable modes are localized at the interface, indicating that these are indeed interface modes.

We compare our results to the case of a continuous, i.e., nonlattice, system. In Ref. [8], it was observed that in the case of a narrow interface, the binary condensate displays the classical dispersion relation [13]

$$
\omega=\sqrt{\frac{\alpha}{2 \rho} k_{e}^{3}-v^{2} k^{2}} \quad \text { (classically), }
$$

where $v$ is the velocity of the unperturbed flow and $\alpha$ is the surface tension. However, if the interface is wider, the upper stability line lies close to the line $\left|k_{e}-k / 2\right|=k$; this was in Ref. [8] termed the countersuperflow instability. Apparently,
Fig. 1 is in this latter regime, and the discreteness is not seen to cause considerable deviations from the linear stability line.

We note a few curious features in Fig. 1. For very small $k$, the instability seems to be absent; this will be discussed further in Sec. III B. For wave vectors exceeding a critical value, $k>k_{c}=\pi / 2$, it is seen in Fig. 1 that all excitation wave vectors become unstable. This is no longer an interface mode, but an instability known to occur also in single-component discrete BECs [15]. The physics behind the instability is that the single-particle dispersion relation, $\epsilon_{k}=J(1-\cos k)$, has an inflection point at $k_{c}$, and thus the effective mass goes from positive to diverging to negative. This is well understood and studied in single-component BECs, and we will not dwell on it further, except to conclude that the interesting range of $k$ values for the case $\left(k_{1}, k_{2}\right)=(0, k)$ ranges between $-\pi / 2$ and $\pi / 2$.

To illustrate the long-time behavior of Kelvin-Helmholtz instability, we simulate the time development of the discrete GP equation. A series of snapshots are shown in Fig. 2 for the case $k=5 \pi / 64 \approx 0.245$. The time development is simulated using a split-step Fourier technique, with a grid of size $64 \times 64$ sites and periodic boundary conditions along both directions. For the chosen wave vector $k=5 \pi / 64$, a surface wave with a dominant wave vector $k_{e}=3 \pi / 64$ can be seen to grow (a)

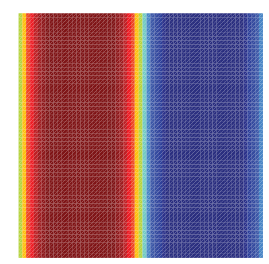

(c)

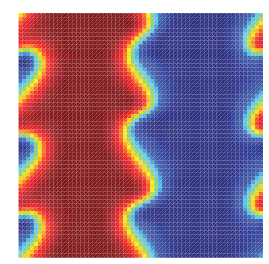

(e)

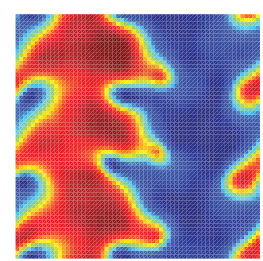

(g)

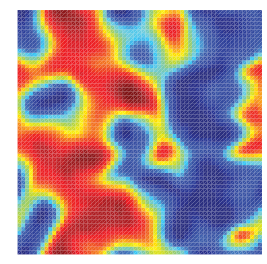

(b)

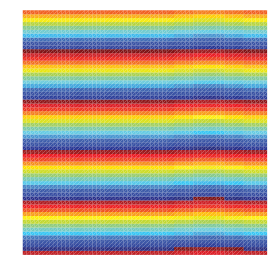

(d)

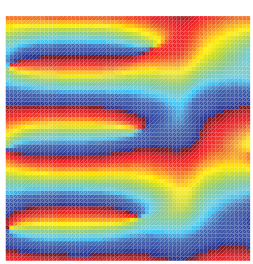

(f)

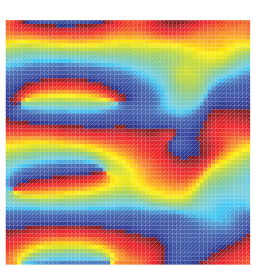

(h)

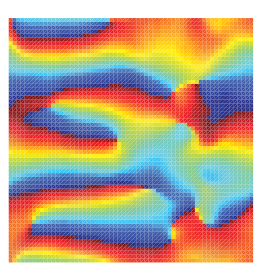

FIG. 2. (Color online) Time development of the instability for the case $k=5 \pi / 64$, with the remaining parameter values as in Fig. 1 . (a), (c), (e), and (g) The density of the component 1, where red indicates high density and blue is zero density; (b), (d), (f), and (h) the corresponding phase. Snapshots are taken at times $t=44$ for (a) and (b), $t=176$ for (c) and (d), $t=265$ for (e) and (f), and $t=400$ for $(\mathrm{g})$ and $(\mathrm{h})$. The field of view is $64 \times 64$ lattice sites. 


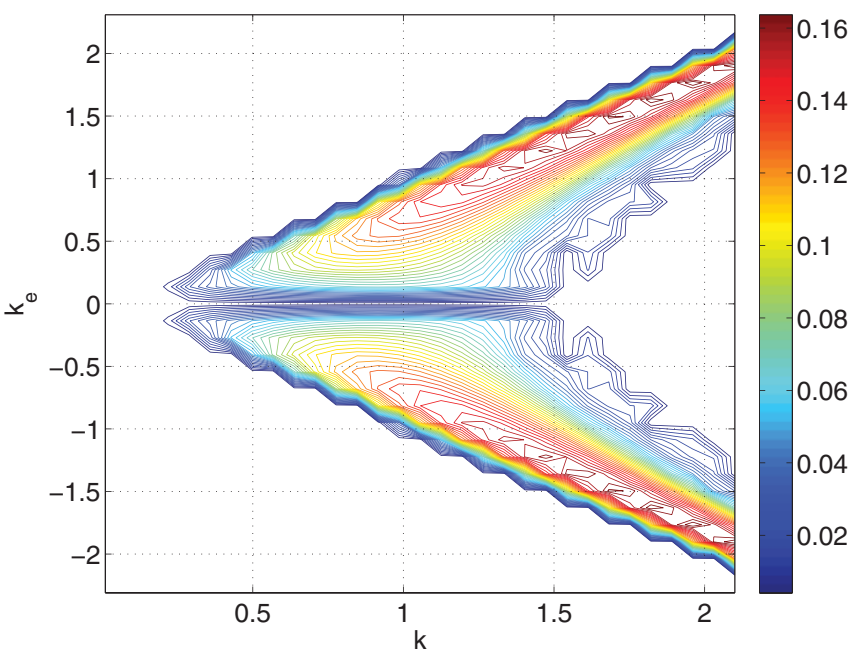

FIG. 3. (Color online) Largest imaginary part of the excitation frequency as in Fig. 1, but with counterflowing condensates having wave vectors $(k / 2,-k / 2)$. The lattice has 256 sites, parameter values are $J / U=1, U_{12} / U=1.1$, and the asymptotic density is $\bar{n}=1$.

exponentially until the nonlinear stage is reached, where the condensates break up into smaller fragments.

For the case of counterflow, $(k / 2,-k / 2)$, the result for the imaginary parts of the modes is shown in Fig. 3. By definition of $k$, the critical relative wave vector for negative effective mass is in this case $k_{c}=\pi$. For large enough $k$, it is seen that the modes with small wave vectors $k_{e}$ are actually stabilized. We thus define a second critical wave number for the flow $k_{s}$ above which long-wavelength excitations are stabilized. The corresponding flow velocity is denoted $v_{s}$. From Fig. 3, we read $k_{s} \approx 1.5$ (the closeness to $\pi / 2$ is suggestive but, in fact, a coincidence). This is a counterpart to the classically known stabilization of long wavelengths when the flow velocity exceeds $\sqrt{8}$ times the speed of sound $c$ [14],

$$
v_{s}=\sqrt{8} c \quad \text { (classically). }
$$

However, in the discrete case, there seems to be no simple relationship between the onset of long-wavelength stability and speed of sound. In this particular case, the flow velocity in each component is $v_{s}=2 J \sin \left(k_{s} / 2\right) \approx 1.4 U$ in dimensionless units, whereas the speed of sound is $c=\sqrt{J U \bar{n}}=U$, but further numerical experimentation shows only a weak dependence of $v_{s}$ on $c$; this puzzle will be resolved in Sec. IV.

Since the system lacks Galilean invariance, the spectrum for a given flow wave vector $k$ is not invariant under a transformation $\left(k_{1}, k_{2}\right) \rightarrow\left(k_{1}-q, k_{2}+q\right)$. We plot as an example in Fig. 4 the imaginary frequency for the same parameters as above for two choices of $k$, comparing the cases $(k, 0)$ and $(k / 2,-k / 2)$. It is seen that the curves are similar, but the symmetric case $(k / 2,-k / 2)$ is more unstable in the sense that it has a higher maximum imaginary frequency. Further numerical experimentation (not shown here) indicates that this observation holds quite generally.

\section{B. Narrow interface physics: Effects of discreteness}

Figure 5 shows the stability spectrum of a system at a higher density, $\bar{n}=4$, and a stronger repulsion between the components, $U_{12}=1.5 U$. The combined effect of increased density, which means larger interaction energy, and increased interspecies repulsion implies a narrower interface [16]. Already in the case $\bar{n}=1, U_{12}=1.1 U$, we saw that low flow velocities appeared to be stable, and here the effect is more pronounced. This feature of the instability is not seen in simulations of homogeneous BECs [7,8] or in classical fluids in the absence of external forces [13]. We attribute this to the discrete density profile at the interface, as will be detailed below.

In Fig. 5, we note also that there is a stable region for large flow wave vectors $k>k_{s}$, just as in the case $\bar{n}=1$, but (a)

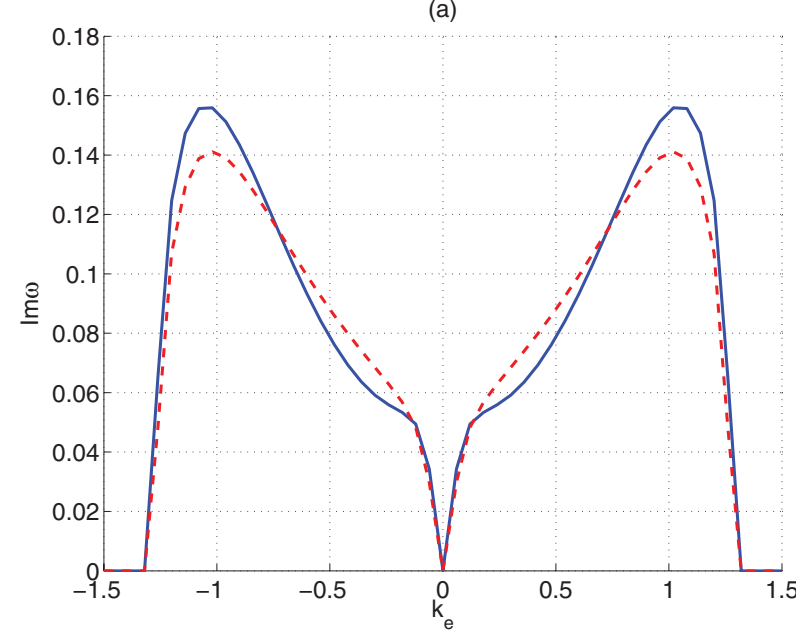

(b)

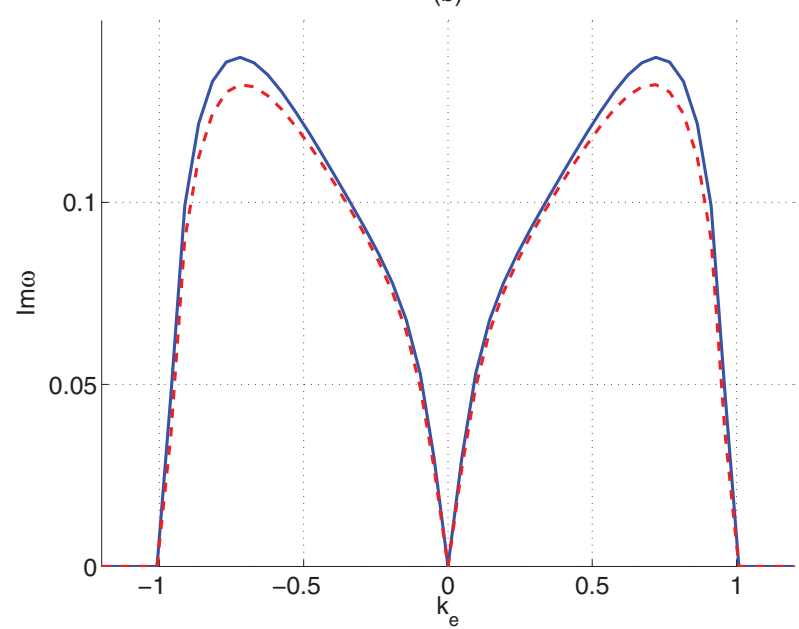

FIG. 4. (Color online) Comparison of the largest imaginary part of the excitation frequency as a function of the excitation wave number $k_{e}$ in the cases of one flowing component, $(k, 0)$ (dashed red line), and counterflow, $(k / 2,-k / 2)$ (solid blue line). Parameter values are as in Fig. 1, but the wave vector is fixed at $k=1.3$ in (a) and $k=1.0$ in (b). For the case of one flowing component, the $k_{e}$ axis is shifted by $k_{e} \rightarrow k_{e}-k / 2$. The lattice has 256 sites, parameter values are $J / U=1, U_{12} / U=1.1$, and the asymptotic density is $\bar{n}=1$. 


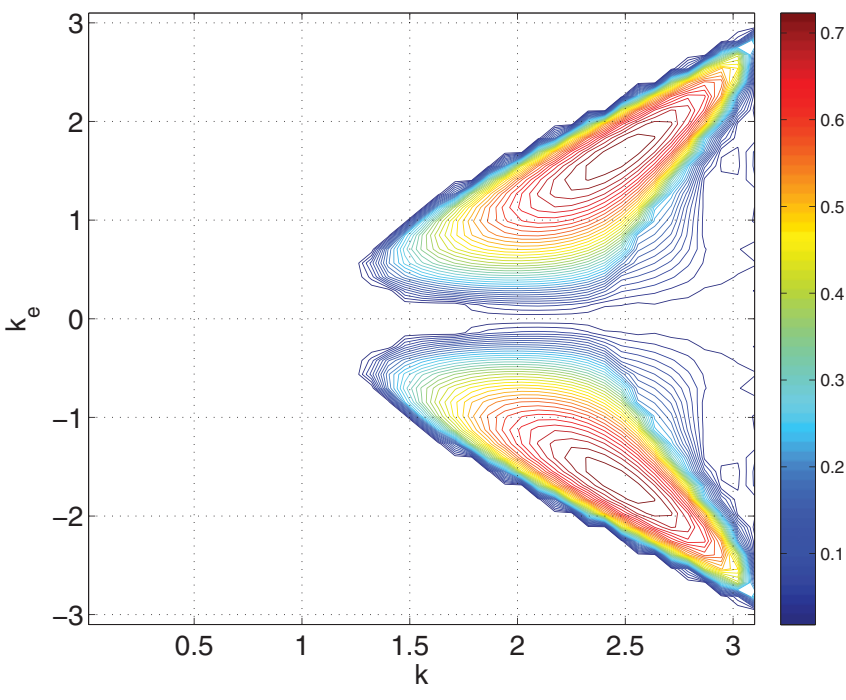

FIG. 5. (Color online) Growth rate of the dynamically most unstable mode (i.e., the imaginary part of the mode with the largest imaginary frequency) as a function of flow wave vector $k$ and and mode wave vector $k_{e}$. Here both components are in motion with counterdirected wave vectors $(k / 2,-k / 2)$. The parameters are $J / U=1, U_{12} / U=1.5$, and the asymptotic density is $\bar{n}=4$.

now $k_{s} \approx 2.8$. We will return to this in Sec. IV. Finally, we observe that the upper instability line at large $k$ values is still approximately linear with unit slope, indicating that we are in the countersuperflow regime despite the narrower interface.

We now turn to the stability of slow flow wave vectors $k$. First, recall how a dynamical instability in the Bogoliubov equations results from a collision between a quasiparticle mode and a quasihole mode [17]. Starting from a reference point in phase space (here take $k=0$ ), we consider a quasiparticle mode $\mathbf{v}_{a}$ and a quasihole mode $\mathbf{v}_{b}$ with real, nearly degenerate eigenfrequencies $\omega_{0 a}$ and $\omega_{0 b}$. We then slightly increase a control parameter (in this case, $k$ ) and thus transform the Bogoliubov matrix $M \rightarrow M^{\prime}=M+\delta M$. Writing $\mathbf{v}=a \mathbf{v}_{a}+b \mathbf{v}_{b}$ and solving the Bogoliubov equations for the new eigenfrequencies $\omega$, we find in the general case

$$
\begin{aligned}
\omega= & \frac{\omega_{0 a}+\omega_{0 b}+\delta \omega_{a}+\delta \omega_{b}}{2} \\
& \pm \sqrt{\left(\frac{\omega_{0 a}-\omega_{0 b}+\delta \omega_{a}-\delta \omega_{b}}{2}\right)^{2}-|X|^{2}},
\end{aligned}
$$

where $\delta \omega_{a}=\mathbf{v}_{a}^{\dagger} \delta M \mathbf{v}_{a}, \delta \omega_{b}=-\mathbf{v}_{b}^{\dagger} \delta M \mathbf{v}_{b}$, and $X=\mathbf{v}_{a}^{\dagger} \delta M \mathbf{v}_{b}$. In other words, when the two modes become degenerate, the cross-term makes them unstable.

In the present case, we consider two modes related by the symmetry of Eq. (21); thus, we have $\omega_{0 a}+\delta \omega_{a}=-\omega_{0 b}-$ $\delta \omega_{b}$, and

$$
\omega= \pm \sqrt{\left(\omega_{0 a}+\delta \omega_{a}\right)^{2}-|X|^{2}}
$$

In a homogeneous, i.e., nondiscrete system, the lowest-lying surface mode corresponds to a uniform translation of the boundary and is thus a Goldstone mode; at $k=k_{e}=0$ it has zero energy, i.e., $\omega_{0 a}=0$ in the limit $k_{e}=0$. With increasing $k_{e}$, its energy increases from zero. A finite $k$ results in a
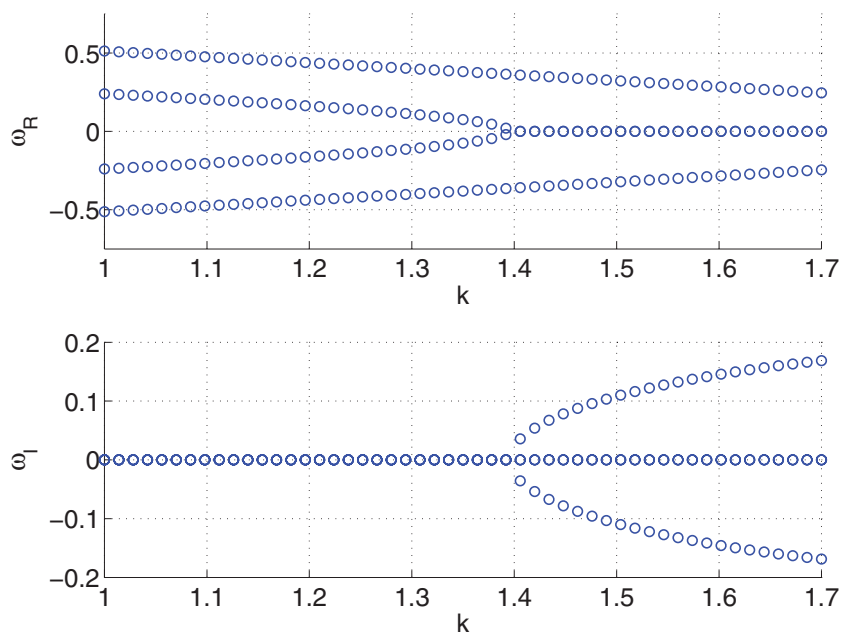

FIG. 6. (Color online) A few Bogoliubov eigenvalues for a fixed excitation wave vector $k_{e}=0.3$ as functions of the flow wave vector $k$. Parameters are as in Fig. 5. (top) Real parts and (bottom) imaginary parts.

shift in the Bogoliubov matrix $\delta M$ as discussed above, and the Kelvin-Helmholtz instability comes about because $|X|$ is always greater than $\delta \omega_{a}$ in these systems. The onset of instability therefore tends toward $k=0$ as $k_{e}$ tends to zero.

In a discrete system, however, translational symmetry is lost, and there is no Goldstone mode: A small shift of the boundary profile costs a finite amount of energy. This translates into $\omega_{0 a}=\epsilon>0$ at $k_{e}=0$, which in turn creates a threshold value of $k$ for the onset of instability. For stronger interactions or higher densities, the interface is thinner, and the effects of discreteness are more pronounced. This explains the increased threshold value for higher density.

Figure 6 displays some of the lowest-lying Bogoliubov eigenvalues for a fixed $k_{e}=0.3$ as functions of $k$ at $\bar{n}=4$. It is clearly seen how instabilities result from a merging of two eigenstates with equal but opposite energies, thus related by the symmetry in Eq. (21). For large $k$ similar instabilities happen for many other modes, and here, for clarity, we plot just the appearance of the first instability. The modes responsible for the dominant instability start out at a small but finite energy for $k=0$; this energy does not tend to zero as $k_{e} \rightarrow 0$ (although the latter fact cannot be inferred from Fig. 6). The mode functions $u_{i}, v_{i}$ are plotted in Fig. 7. These are the lowest-lying surface modes corresponding to translation of the surface; notice how the symmetry (21) is manifest in the plots.

\section{TOWARD AN ANALYTICAL DESCRIPTION}

In order to obtain a feeling for the mathematics and physics behind the Kelvin-Helmholtz instability, we show here a heuristic derivation of an approximate analytical formula for the excitation frequencies. Some related research has also been done by Ruostekoski and Dutton [18]. In effect, we replace the spatially dependent coefficients in Eq. (17) by $c$ numbers. This can be justified by expanding the solution $\mathbf{v}$ in a suitable basis [19]. It can also be affected by simply assuming a local-density approximation at the center of the interface. We discuss the more symmetric case with counterdirected 
(a)

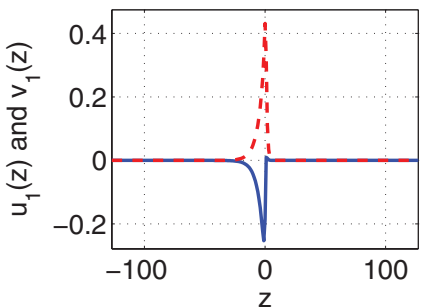

(c)
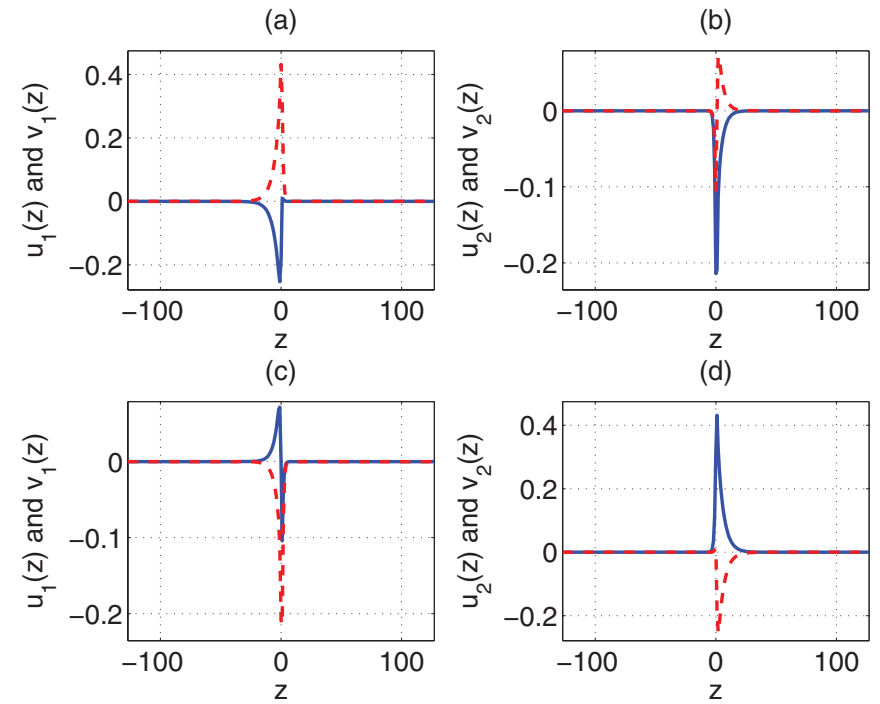

(d)

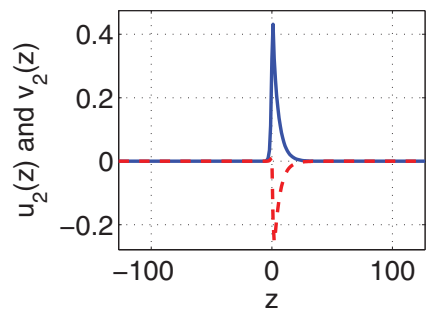

FIG. 7. (Color online) Bogoliubov eigenstates for the lowestlying surface modes, functions $u_{j}(z)$ (blue solid lines) and $v_{j}(z)$ (red dashed lines) at $k_{e}=0.3$ and $k=1.25$, which is below the instability threshold. (a) and (b) Quasiparticle mode; (c) and (d) quasihole mode. (left) Mode functions $u_{1}(z), v_{1}(z)$ associated with component 1 and (right) mode functions $u_{2}(z), v_{2}(z)$. The lattice has 256 sites, parameter values are $J / U=1, U_{12} / U=1.5$, and the asymptotic density is $\bar{n}=4$.

currents, $\left(k_{1}, k_{2}\right)=(k / 2,-k / 2)$, since it allows for solutions on a closed form. The matrix is approximated as

$\hat{M}=\left(\begin{array}{cccc}\epsilon+\Delta_{+}+g & g & C & C \\ g & \epsilon+\Delta_{-}+g & C & C \\ C & C & \epsilon+\Delta_{-}+g & g \\ C & C & g & \epsilon+\Delta_{+}+g\end{array}\right)$,

where $g$ is proportional to the in-species interaction energy and $C$ is a measure of the interspecies repulsion. $\epsilon$ is a self-energy associated with bending in the $z$ direction of the respective mode function, which is to be identified with the finite energy $\omega_{0 a}$ of the almost-Goldstone modes discussed above. Finally, $\Delta_{ \pm}$are the relative kinetic energies. In the discrete lattice case,

$$
\Delta_{ \pm}=-2 J(1-\cos k / 2)+2 J\left[1-\cos \left(k_{e} \pm k / 2\right)\right],
$$

and in the continuous case without a lattice,

$$
\Delta_{ \pm}=\frac{\hbar^{2}}{2 m}\left(k_{e}^{2} \pm k_{e} k\right) .
$$

The continuous case is simplest: Inserting the dispersion, putting $\hbar=m=1$ for convenience, we obtain the eigenfrequencies

$$
\begin{aligned}
\omega^{2}= & \left(2 g+\frac{1}{2} k_{e}^{2}+\epsilon\right)\left(\epsilon+\frac{1}{2} k_{e}^{2}\right)+\frac{1}{4} k_{e}^{2} k^{2} \pm 2 \sqrt{\epsilon+\frac{1}{2} k_{e}^{2}} \\
& \times \sqrt{\left(2 g+\epsilon+\frac{1}{2} k_{e}^{2}\right) \frac{1}{4} k_{e}^{2} k^{2}+C^{2}\left(\epsilon+\frac{1}{2} k_{e}^{2}\right)} .
\end{aligned}
$$

This equation is of the same form as that in Ref. [8], describing the countersuperflow instability. Clearly, the case with a minus sign lets $\omega^{2}$ become negative for certain parameter values and thus describes the unstable modes. The discrete case yields somewhat clumsier expressions but can be solved; we discuss the differences below.

The choice of parameters can be done with the benefit of hindsight; however, assuming a local-density approximation in the midpoint of the interface, where, in the limit of a broad interface, the densities $n_{1}$ and $n_{2}$ are half their bulk values, we readily obtain $g=U \bar{n} / 2=\mu / 2$ and $C=$ $U_{12} \bar{n} / 2=\left(U_{12} / U\right) g$ (note a factor 2 difference compared to the definitions in Ref. [8]). The self-energy $\epsilon$ is smaller than the other energies and so is $C-g$, so we first put $\epsilon=0$ and $C=g$ in Eq. (28) and find the points where $\omega^{2}$ becomes negative; these are the boundaries for instability. We obtain the solutions

$$
\begin{aligned}
k_{e} & =0, \\
k & =k_{e}, \\
k^{2}-k_{e}^{2}-4 \mu & =0 .
\end{aligned}
$$

Linearizing around the first instability, we obtain

$$
\omega^{2}=\frac{1}{2} k_{e}^{2}\left[\frac{1}{2} k^{2}+\mu-\sqrt{\mu\left(\mu+2 k^{2}\right)}\right]+\mathcal{O}\left(k_{e}^{4}\right),
$$

which is the well-known linearly increasing imaginary frequency, which exists for $k^{2}<4 \mu$. The modes are stabilized again when $k_{e}>k$. The upper stability limit lies at $k_{e}=k$, as observed in Ref. [8]. However, when $k^{2}>4 \mu$, small wave vectors $k_{e}$ are stabilized. This is similar to the well-known classical stabilization at relative flow velocities exceeding $\sqrt{8}$ times the sound velocity, as we discussed in Sec. III A [14]. However, even in the continuous case, the prefactor does not match the classical result (or Ref. [8]); we simply attribute this to the quite different kind of dispersion relation.

A small but finite $\epsilon$ will introduce small corrections to the above. In order to salvage the linear dispersion at small excitation wave vectors $k_{e}$, which is observed to be the case numerically, we must put $C=g+\epsilon / 2$. With a finite $\epsilon$, slow flow velocities are stabilized. This happens only in the discrete case, but in order to bring out the qualitative features while displaying reasonably compact formulas, we show here the bastardized expression resulting from using a continuous-case dispersion relation together with a finite self-energy. Expanding the eigenfrequency in powers of $k$ and $k_{e}$, one obtains

$$
\omega^{2}=\frac{1}{2} k_{e}^{2}\left(\frac{1}{2} k_{e}^{2}+\epsilon\right)-\frac{1}{4} k^{2} k_{e}^{2}+\mathcal{O}\left(k^{6}, k_{e}^{6}, k^{2} k_{e}^{4}, k^{4} k_{e}^{2}\right),
$$

which is positive for $k^{2}<q^{2}+2 \epsilon$. Thus, slow flow velocities are seen to be absolutely stable. Classically, this only happens in the presence of gravity or an equivalent asymmetric force $[7,13]$, but in the discrete system, there is stabilization even in the absence of forces.

The discrete-case dispersion relation leads to more complicated algebra, but all the qualitative conclusions remain the same. When $\epsilon=0$ and $g=C$, the frequency is again zero for $k_{e}=0$ and $k_{e}=k$; a finite $\epsilon$ stabilizes small $k$. The most important modification is that of the sound-speed limit, where we now obtain stability at small $k_{e}$ if $k>k_{s}$, with $k_{s}$ given by

$$
2 J \cos \left(\frac{k_{s}}{2}\right)=-\mu / 2+\sqrt{\mu^{2} / 4+4 J^{2}},
$$


whose solution is always real. The continuous-case expression is recovered when $\mu / J$ is small; then the flow velocity is $v_{s}=2 J \sin k_{s} \approx \sqrt{4 \mu}$, similar to the continuous case.

Furthermore, for $k>\pi / 2$, the spectrum changes character completely, and a wide range of excitation wave vectors $k_{e}$ becomes unstable; this is again a manifestation of the bulk instability associated with negative effective mass of Ref. [15].

Thus, a heuristic constant-amplitude calculation has managed to bring out all the features present in the numerical results. The familiar linear increase of imaginary frequency at small $k_{e}$ for a given flow wave vector $k$, the stabilization at excitation wave vectors $k_{e}$ exceeding $k$, the absolute stabilization of slow relative velocities, the stabilization of small $k_{e}$ for $k$ above a cutoff depending on the sound velocity, and even the bulk instability when the effective mass becomes negative could all be described.

\section{STRONGLY CORRELATED REGIME}

The discussion so far has been limited to the case of two Bose gases in the Bose-Einstein-condensed state. This is the state of the system when the ratio $J / U$ is not very small and when the density is not very small. In the general case, one must account for quantum fluctuations whose most striking effect is to drive a quantum phase transition between the superfluid and Mott-insulating phases [9]. In addition, quantum fluctuations are seen to shift the transition line between mixed and phasesegregated phases, as we shall see below.

The many-body Hamiltonian (1) has been seen to display a rich phase diagram $[10,11]$. In Fig. 8, we display the portion of greatest interest for our purposes. The phase diagram is calculated in the Gutzwiller approximation, which is explained below. The general picture is that for weak enough hopping $J$, except in degenerate cases, both gases are in the Mott-insulating state (marked MI in Fig. 8) with suppressed on-site number fluctuations and zero superfluid

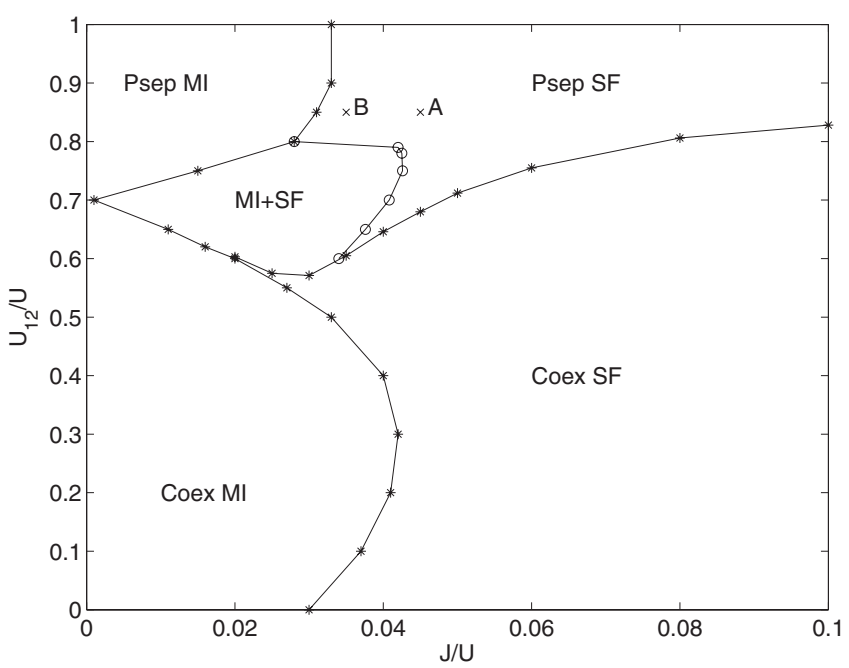

FIG. 8. Phase diagram for a mixture of two identical Bose gases, calculated in the Gutzwiller approximation. The chemical potential is taken to be $\mu=0.7 U$. Points $A$ and $B$ mark the parameters for which the two simulations described in the text are done. density; conversely, for large enough $J$ the bosons are always superfluid (SF). If the intercomponent interaction $U_{12}$ is large enough compared with the chemical potential $\mu$, then the two components are phase separated (marked Psep), either Mott insulating or superfluid; and if $U_{12}$ is small enough or $\mu$ is big enough, the two components coexist (marked Coex). There exists one further phase close to the line $U_{12}=\mu$ at weak hopping, where one component is Mott insulating and the other one is superfluid with a lower density (marked $\mathrm{SF}+\mathrm{MI}$ in the phase diagram). Finally, note that for a pair of Bose-Einstein-condensed gases, the transition between phase-separated and coexisting phases takes place at $U_{12}=U$. In contrast, in the limit of weak $J$, phase separation takes place at $U_{12}=\mu$.

The Gutzwiller approximation is based on a mean-field ansatz for the many-body state,

$$
\left|\psi_{G}(t)\right\rangle=\prod_{r} \prod_{j=1}^{2}\left|\phi_{r, j}(t)\right\rangle .
$$

This ansatz amounts to treating both the hopping between sites and the interspecies interaction in a mean-field fashion. This can be done since on-site entanglement between the two species is not expected to be important. For computations, it is convenient to expand the on-site states in a local Fock basis with an upper cutoff $n_{\max }$,

$$
\left|\phi_{r, j}(t)\right\rangle=\sum_{n=0}^{n_{\max }} C_{r, j, n}(t)|n\rangle_{r, j} .
$$

In the Gutzwiller approximation, the local total density $n$ and condensate wave function for each of the components are readily computed. We characterize the system by studying the behavior of local density $n_{j}$, the local condensate density $n_{c j}=\left|\Phi_{j}\right|^{2}$, and phase $\varphi_{j}=\arg \Phi_{j}$. In order to calculate the ground state, the Hamiltonian (1) is minimized with respect
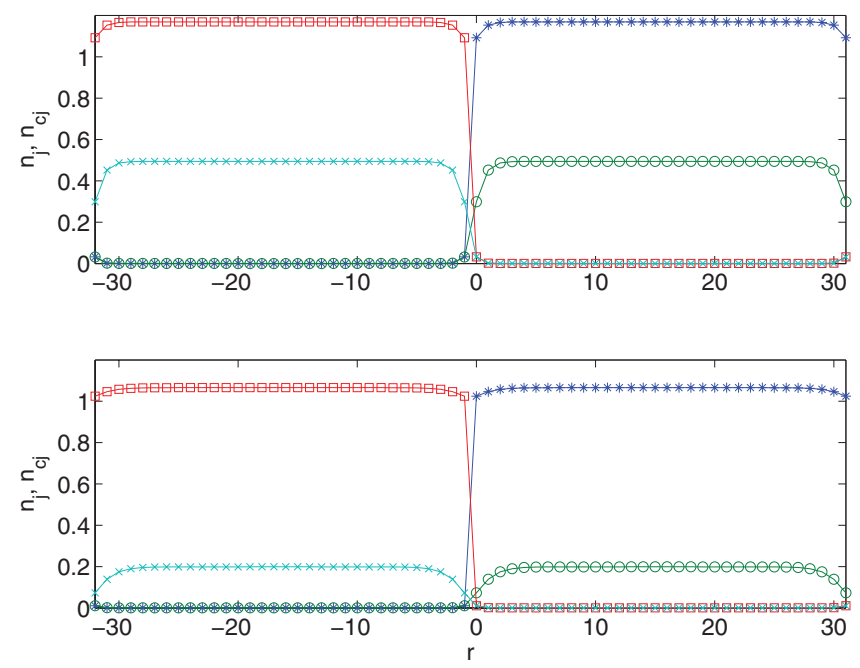

FIG. 9. (Color online) Cross sections of the density profile of two phase-segregated Bose gases at points (top) $A$ and (bottom) $B$ in the phase diagram. Squares denote the density for component $1 n_{1}$, crosses denote $n_{c 1}$, asterisks $n_{2}$, and circles denote $n_{c 2}$. Lines are to guide the eye. 
to the complex coefficients $C_{r, j, n}$. To simulate dynamics, we propagate the coupled equations of motion [20,21]

$$
i \frac{\partial C_{r, j, n}}{\partial t}=\frac{\delta}{\delta C_{r, j, n}^{*}}\left\langle\psi_{G}(t)|H| \psi_{G}(t)\right\rangle .
$$

For the present simulation of Kelvin-Helmholtz physics, the initial condition was constructed by calculating the phase-separated ground state, then imprinting counterdirected currents on the two components, and finally adding a small amount of random noise onto each of the complex coefficients $C_{r, j, n}$.

We simulate interface dynamics in the Psep SF phase. In the Mott-insulating phase, dynamics is quite trivially absent. In the $\mathrm{SF}+\mathrm{MI}$ phase, there is also no interface dynamics: Since the Mott-superfluid phase transition is second order, there is no surface tension, and hence no capillary waves are expected to form between superfluid and Mott-insulating regions. Phenomena connected to the melting of Mott insulators are conceivable at high enough energies (cf. [21,22]), but we will not pursue this here.

The first simulation is done at point $A$ in the phase diagram of Fig. 8: $J=0.045 U, U_{12}=0.85 U$, and $\mu=0.7 U$. Here we have two phase-separated superfluids, and as seen in Fig. 9, the Bose-Einstein-condensed part of the fluid is less than half the total density. Also the superfluid density is expected to be small close to the Mott transition [23]. Figure 10 shows the dynamics of a system with symmetrically imprinted wave vectors $(k / 2,-k / 2)$, where $k=7 \pi / 16 \approx 1.3744$. It is seen that the condensate densities behave very much like in the pure-condensate case. The time scale is vastly different due to the difference in tunneling matrix element $J$. In addition, it is seen that the total density is affected very little, and on the time scales we have simulated the system seems to be settling down into a phase-separated steady state. The two fluids do not mix; the main effect of the $\mathrm{KH}$ instability is to excite vortices within the respective components. In the phase plots one can see vortices as phase singularities; they show up as zeros of the (a)

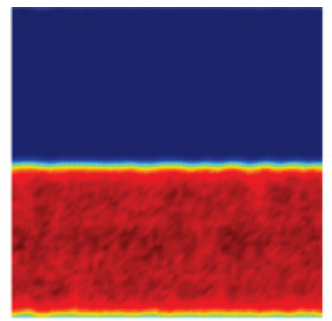

(d)

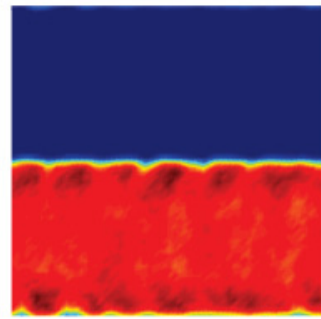

(g)

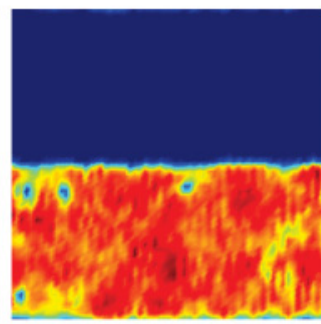

(j)

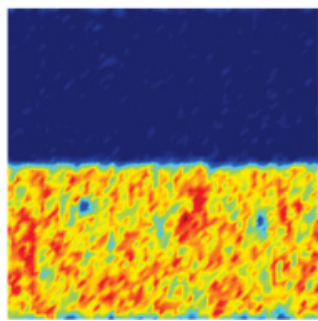

(b)

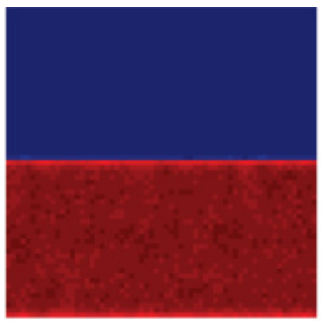

(e)

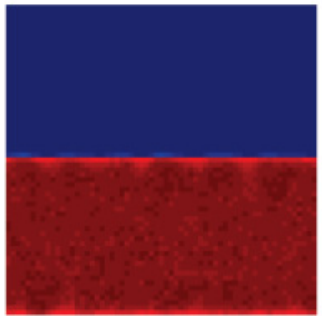

(h)

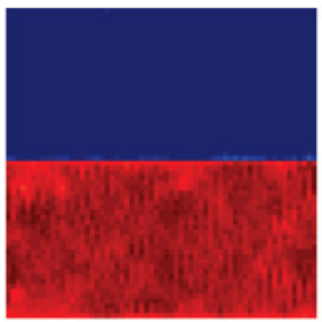

(k)

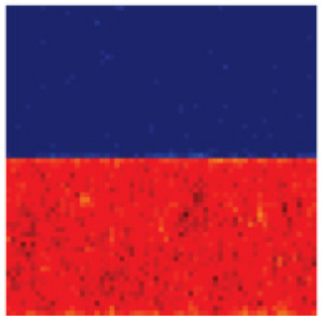

(c)

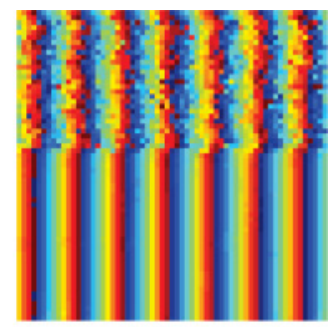

(f)

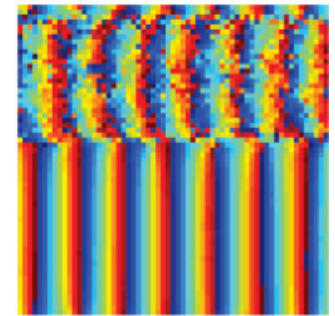

(i)

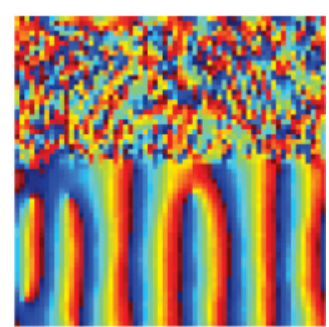

(I)

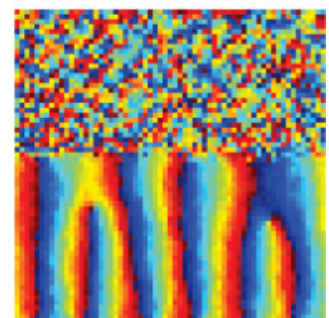

FIG. 10. (Color online) Time development in the Gutzwiller approximation of a system with $J=0.045 U, U_{12}=0.85 U$, and $\mu=0.7 U$, with wave vectors $(k / 2,-k / 2)$, where $k=7 \pi / 16$. Snapshots displayed in the different rows are taken at times (from top to bottom) $100 / U$, $200 / U, 300 / U$, and $4000 / U$. (left) Condensate density $n_{c}$, (middle) total density $n$, and (right) the phase $\varphi$ of bosons of component 1 . The corresponding quantities for component 2 are not shown but are similar to the ones shown mirrored in the $x$ axis. The field of view is $64 \times 64$ lattice sites. 
(a)

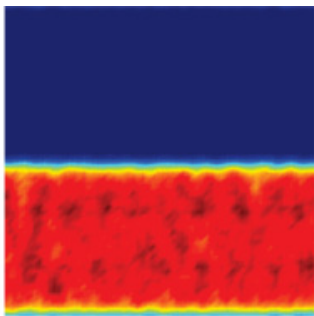

(d)

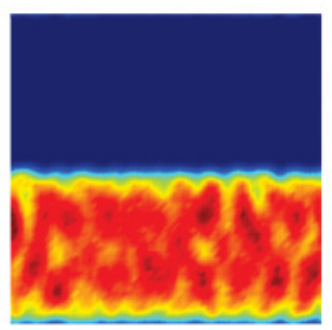

(g)

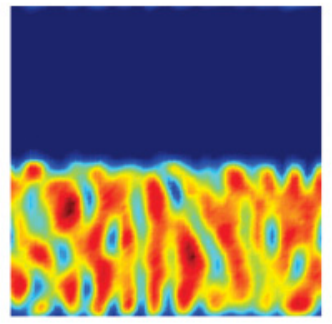

(j)

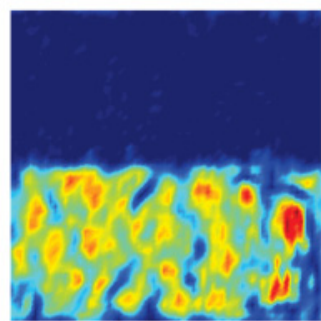

(b)

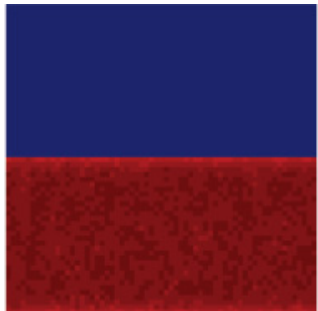

(e)

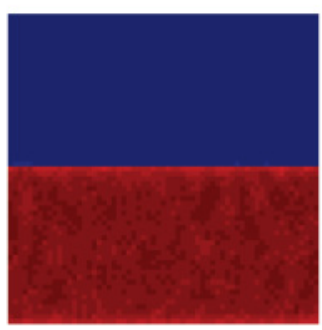

(h)

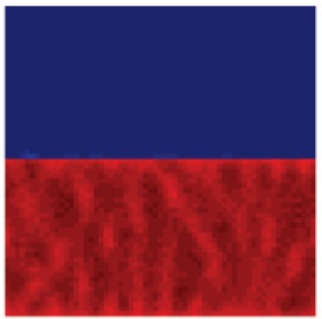

(k)

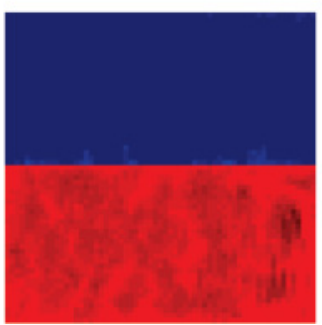

(c)

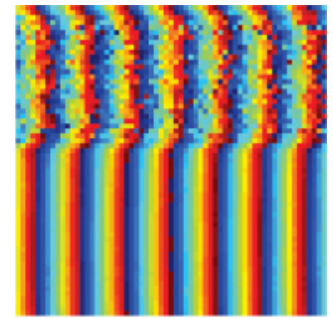

(f)

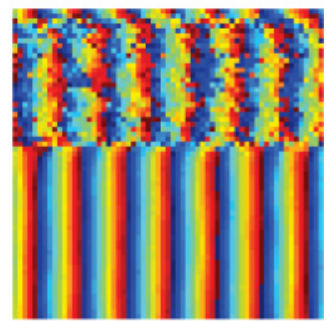

(i)

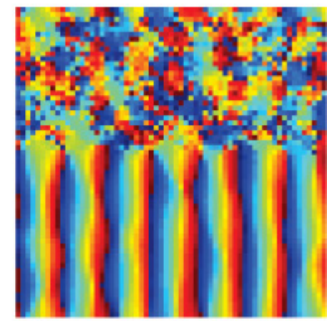

(l)

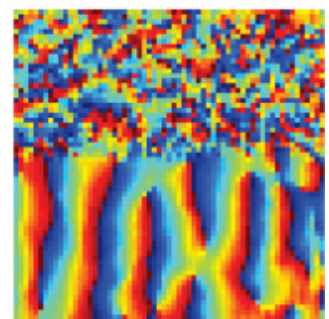

FIG. 11. (Color online) Time development in the Gutzwiller approximation of a system with $J=0.035 U, U_{12}=0.85 U$, and $\mu=0.7 U$, with wave vectors $(k / 2,-k / 2)$, where $k=7 \pi / 16$. Snapshots displayed in the different rows are taken at times (from top to bottom) $100 / U$, $200 / U, 300 / U$, and $4000 / U$. (left) Condensate density $n_{c}$, (middle) total density $n$, and (right) the phase $\varphi$ of bosons of component 1 . The corresponding quantities for component 2 are not shown but are similar to the ones shown mirrored in the $x$ axis. The field of view is $64 \times 64$ lattice sites.

condensate density, but the total density is not depleted. These are vortices filled with Mott-insulating atoms, as previously described in Refs. [24,25].

We next show a simulation at point $B$ in the phase diagram of Fig. 8: $J=0.035 U, U_{12}=0.85 U$, and $\mu=0.7 U$. This is still in the regime of two phase-separated superfluids, but even closer to the Mott transition. Figure 11 shows the dynamics. We see that the dynamics is, in fact, more rapid here than in the case of larger $J$. At times $t=200 / U$ and $t=300 / U$, a significant wave pattern develops in the condensate component, and at $t=1000 / U$, the system contains many more vortices than in the case in Fig. 10. The total density is less affected but exhibits some protruding features at the surface. Clearly, with a smaller condensate fraction, the threshold energy is smaller for modulation of condensate density and formation of vortices. Close to the transition, where the condensate density decreases rapidly toward zero, this overcomes the effect of the modest reduction of $J$.

\section{CONCLUSIONS}

In summary, we have studied the Kelvin-Helmholtz instability on the interface between two lattice Bose gases in relative motion. The instability is seen to be affected by three effects introduced by the lattice potential: broken translational symmetry, discreteness, and quantum fluctuations. Broken translational symmetry affects the instability in such a way that the excitation frequencies do not depend only on the relative velocity, but rather on the flow velocities of the two gases separately; the symmetric case of two counterflowing gases is seen to be the most unstable situation. Second, the discreteness will stabilize slow relative currents, so that the instability is prevented if the relative velocity is low enough. Finally, strongly correlated physics affects the physics in such a way that a neighboring Mott-insulating phase will prevent the two Bose systems from mixing after the Kelvin-Helmholtz instability is first excited. Close to the Mott phase transition, 
only the superfluid density will take part in the instability, but the total density will hardly be affected.

The physics we have discussed here could be experimentally probed, for example, in a homogeneous two-component lattice system where the condensates are phase separated. Here a limiting factor would be the time scale for the moving condensates to pass each other. For instabilities to be observable, parameters should be chosen in such a way that instabilities grow faster than the condensates separate. Alternative ways to realize these kinds of systems experimentally might be to have a phase-separated double-condensate system in a trap [7] or in an elongated toroidal trap with one component on top of the other. Such systems would have the benefit of periodic boundary conditions, which allows more time for interface dynamics. Naturally finite-sized systems have the drawback that one cannot explore the physics at wavelengths that are larger than the system size.

\section{ACKNOWLEDGMENTS}

E.L. acknowledges support from the Swedish Research Council (VR). J.-P.M. acknowledges support from the Academy of Finland (Project No. 135646). The authors also acknowledge useful discussions with Prof. Vitaly Bychkov. Calculations have been conducted using the resources of the High Performance Computing Center North (HPC2N). This project was initiated during the NORDITA Program "Quantum solids, liquids and gases."
[1] H. Hasegawa, M. Fujimoto, T.-D. Phan, H. Réme, A. Balogh, M. W. Dunlop, C. Hashimoto, and R. TanDokoro, Nature (London) 430, 755 (2004).

[2] R. Blaauwgeers, V. B. Elstov, G. Eska, A. P. Finne, R. P. Haley, M. Krusius, J. J. Ruohio, L. Skrbek, and G. E. Volovik, Phys. Rev. Lett. 89, 155301 (2002).

[3] G. E. Volovik, JETP Lett. 75, 418 (2002).

[4] D. S. Hall, M. R. Matthews, C. E. Wieman, and E. A. Cornell, Phys. Rev. Lett. 81, 1543 (1998).

[5] J. Stenger, S. Inouye, D. M. Stamper-Kurn, H.-J. Miesner, A. P. Chikkatur, and W. Ketterle, Nature (London) 396, 345 (1998).

[6] G. Modugno, G. Ferrari, G. Roati, R. J. Brecha, A. Simoni, and M. Inguscio, Science 294, 1320 (2001).

[7] H. Takeuchi, N. Suzuki, K. Kasamatsu, H. Saito, and M. Tsubota, Phys. Rev. B 81, 094517 (2010).

[8] N. Suzuki, H. Takeuchi, K. Kasamatsu, M. Tsubota, and H. Saito, Phys. Rev. A 82, 063604 (2010).

[9] D. Jaksch, C. Bruder, J. I. Cirac, C. W. Gardiner, and P. Zoller, Phys. Rev. Lett. 81, 3108 (1998).

[10] S. Powell, Phys. Rev. A 79, 053614 (2009).
[11] M. Iskin, Phys. Rev. A 82, 033630 (2010).

[12] A. L. Fetter, Ann. Phys. (NY) 70, 67 (1972).

[13] S. Chandrasekhar, Hydrodynamic and Hydromagnetic Stability, International Series of Monographs on Physics (Dover, New York, 1961).

[14] R. A. Gerwin, Rev. Mod. Phys. 40, 652 (1968).

[15] L. De Sarlo, L. Fallani, J. E. Lye, M. Modugno, R. Saers, C. Fort, and M. Inguscio, Phys. Rev. A 72, 013603 (2005).

[16] P. Ao and S. T. Chui, Phys. Rev. A 58, 4836 (1998).

[17] E. Lundh and H. M. Nilsen, Phys. Rev. A 74, 063620 (2006).

[18] J. Ruostekoski and Z. Dutton, Phys. Rev. A 76, 063607 (2007).

[19] H. M. Nilsen and E. Lundh, Phys. Rev. A 77, 013604 (2008).

[20] J. Zakrzewski, Phys. Rev. A 71, 043601 (2005).

[21] E. Lundh, Phys. Rev. A 84, 033603 (2011).

[22] K. V. Krutitsky and P. Navez, Phys. Rev. A 84, 033602 (2011).

[23] A. M. Rey, K. Burnett, R. Roth, M. Edwards, C. J. Williams, and C. W. Clark, J. Phys. B 36, 825 (2003).

[24] C. Wu, H. D. Chen, J. P. Hu, and S. C. Zhang, Phys. Rev. A 69, 043609 (2004).

[25] E. Lundh, Europhys. Lett. 84, 10007 (2008). 